\title{
Polysèmes
}

Revue d'études intertextuelles et intermédiales

\section{Collisions et télescopages temporels dans les installations de Fred Wilson}

Collisions and Temporal Clashes in Fred Wilson's Installations

\section{Claudine Armand}

\section{(2) OpenEdition}

Journals

Édition électronique

URL : http://journals.openedition.org/polysemes/1934

DOI : 10.4000/polysemes.1934

ISSN : 2496-4212

Éditeur

SAIT

\section{Référence électronique}

Claudine Armand, «Collisions et télescopages temporels dans les installations de Fred Wilson », Polysèmes [En ligne], 17 | 2017, mis en ligne le 30 avril 2017, consulté le 19 avril 2019. URL : http:// journals.openedition.org/polysemes/1934 ; DOI : 10.4000/polysemes.1934

Ce document a été généré automatiquement le 19 avril 2019

Polysèmes 


\title{
Collisions et télescopages temporels dans les installations de Fred Wilson
}

\author{
Collisions and Temporal Clashes in Fred Wilson's Installations
}

\section{Claudine Armand}

1 L'œuvre que donne à voir et à lire Fred Wilson, né à New York en 1954, est portée par un projet qui s'articule autour des notions de passage, de croisement, d'hybridation de formes et de médiums. Projet sous-tendu par des revendications sociales et politiques et des préoccupations ancrées dans notre monde contemporain. Les questions qui traversent son œuvre sont relatives à l'identité, à l'histoire et à la mémoire, notamment l'histoire du peuple africain américain et, de manière plus générale, aux concepts de perception et de représentation.

2 Fred Wilson est un artiste nomade, collectionneur d'objets glanés au cours de ses voyages ou récupérés et appropriés lors de ses interventions dans les musées, une pratique artistique connue sous le nom de «critique institutionnelle » qu'il développe depuis le début des années 80, à l'instar d'autres artistes de sa génération, comme Andrea Fraser ou Renée Green. Cette pratique s'inscrit dans le sillage des artistes conceptuels qui, dès les années 70 , ont entrepris un travail d'investigation muséale en utilisant diverses stratégies, telles que l'appropriation, la reprise, le détournement et la parodie. Pluridisciplinaire, Fred Wilson manie avec aisance la vidéo, la photographie, le langage verbal qu'il juxtapose et combine dans des configurations souvent déroutantes. L'installation est le dispositif plastique qui lui est cher: ouvert et multidimensionnel, ce dispositif favorise le métissage des genres et la transversalité, le dialogue et la confrontation des hétérogènes. Il suscite également une réflexion sur la frontière entre les arts et sur le rapport du regardeur à l'œuvre, au lieu et au temps. Dans l'espace circonscrit dans lequel déambule le visiteur - parmi les objets, les matériaux et les médiums -, les paramètres temporels sont bousculés. Fred Wilson choisit le plus souvent un espace institutionnel, tel un musée (d'art, d'histoire, d'anthropologie), un site archéologique ou un lieu chargé d'histoire lié à la communauté africaine américaine. Son approche consiste à s'approprier l'espace (intérieur et/ou extérieur), les collections et la 
mémoire encryptée en ces lieux afin d'interroger l'institution, lieu de pouvoir aux multiples ramifications : les rapports hiérarchiques, les modes de fonctionnement, les types de discours qu'ils génèrent et l'effet produit sur le spectateur.

3 Aussi le dispositif plastique, visuel et sonore, est-il indissociable du concept de "dispositif» tel que l'a théorisé Michel Foucault, notamment dans Surveiller et punir: naissance de la prison. Pour lui, le pouvoir est diffus et les hommes sont pris dans des dispositifs, un ensemble hétérogène d'éléments qui s'entrecroisent et les assujettissent (40). En choisissant une méthode d'intervention dans des lieux marqués de rapports sociopolitiques complexes et en privilégiant une approche multidirectionnelle, Fred Wilson démonte et rend visible les mécanismes souvent ténus et imperceptibles de ces lieux de pouvoir et d'autorité qui décident également du sort des artistes issus des minorités, à savoir leur visibilité dans les milieux officiels de l'art.

4 À travers l'agencement spatio-temporel sont ainsi mis en exergue le processus de montage et de démontage plastique et conceptuel, et les modalités d'une pensée critique, celle de l'artiste contemporain face aux institutions. Cette écriture de la déconstruction est manifeste dans le traitement que fait l'artiste du temps, notamment dans la mise en relation et la collision de temps hétérogènes. Au temps sédimenté des fouilles archéologiques se confronte le temps répétitif et démesuré du mythe et du fantasme, le temps linéaire des réalités contemporaines et le temps de l'observation, d'où la question qui consiste à s'interroger sur la nature de l'expérience du temps que fait le spectateur. Le présent article propose d'examiner les collisions et télescopages temporels mis en œuvre par l'artiste dans ses installations, à travers le mouvement de va-et-vient, l'entrecroisement et la confrontation entre passé et présent.

\section{Montage/démontage}

5 Artiste chercheur (archiviste, archéologue, ethnologue), Fred Wilson propose au spectateur, par le procédé du montage/démontage, pris au sens physique et métaphorique du terme, un parcours fragmentaire et saccadé à travers le temps et l'espace. Temps lointain sur le site habité de vestiges de civilisations anciennes, comme dans Untitled (1979), une série de sept photographies en noir et blanc prises en Égypte et au Pérou, ou temps plus proche directement lié à l'histoire du peuple africain américain. En atteste l'expérience que l'artiste a menée en 1994 sous l'égide du centre d'art contemporain de West Salem, en Caroline du nord, intitulée Insight: In Site: In Sight: Incite: Memory, Artist and the Community. Avec une équipe d'archéologues, il a procédé à un travail de fouille dans les fondations de la petite église d'old Salem construite en 1823 pour la communauté noire alors exclue de la congrégation morave. Là, dans les ruines du cimetière, encore appelé le cimetière de l'étranger ("the Stranger's Graveyard») attenant l'église, furent découvertes en 1994 trente et une tombes ${ }^{1}$. Sur ce site, autre lieu de pouvoir et à partir de quelques fragments puisés sur place, Fred Wilson a construit une installation fluide et multiforme débordant du cadre institutionnel, visible à l'extérieur et à l'intérieur de l'église et dans le centre d'art contemporain de la ville. Sur les bancs de l'église aux murs dégradés par l'humidité et le salpêtre, il a reconstruit un village en bois en miniature, plaçant des maquettes de bâtiments importants pour la communauté noire autrefois démolis. Non loin de là, le spectateur pouvait voir des gravats et des tombes portant les noms des défunts, autrefois enfouies, à présent recouvertes de plexiglas. Traversé par le corps de l'artiste et par celui du spectateur et, sous un regard 
contemporain, le site exploré dans les strates géologiques et temporelles du sol (Fred Wilson aime répéter le terme « layers » en parlant du processus de création) prenait ainsi une autre signification, devenant processus et expérience temporaire, sorte de mémorial érigé à la mémoire des marginalisés et des oubliés de l'histoire. Par ce geste de fouille dans l'histoire et la mémoire d'un lieu donnant accès à l'invisible, Fred Wilson opérait symboliquement un déplacement de la périphérie vers le centre.

6 Autre lieu d'expérimentation et d'exploration: le musée. Espace d'exposition et d'archivage, il est avant tout pour l'artiste un lieu dépositaire de mémoire. Son intervention dans les locaux de la Historical Society de Baltimore (considérée alors comme le lieu le plus conservateur de la ville) en 1992 intitulée Mining the Museum, fortement controversée à l'époque, est une œuvre novatrice qui l'a propulsé au devant de la scène artistique américaine. Curateur de l'exposition, le but de Fred Wilson était de mimer les modes de représentation et la rhétorique caractéristique du musée afin d'en révéler les modes de fonctionnement, la rhétorique sous-jacente et de montrer en quoi les choix opérés par l'institution hiérarchisée et les pratiques muséales affectent et orientent la perception du spectateur. Comme le suggère le verbe «Mining ", le geste de l'artiste consistait à s'approprier le lieu pour mieux le sonder dans ses profondeurs, littéralement ouvrir les archives poussiéreuses du musée afin d'en ébranler les fondations et le sortir de sa torpeur ${ }^{2}$. Pour ce faire, il a entrepris un travail d'observation minutieuse et de sélection dans les collections du musée où il a puisé toutes sortes d'objets et d'artefacts, en particulier des objets qui n'avaient jamais été exposés auparavant. Dans cette exposition occupant plusieurs salles, l'exemple le plus frappant est peut-être cette paire de menottes rouillées avec sa clé (datées 1793-1872), placées au milieu d'un service à café en argent repoussé (1830-80) ayant appartenu à une famille bourgeoise de Baltimore, le tout serti dans une vitrine en verre. Plus imposant encore, dans un coin d'une pièce au mur de couleur rouge sur lequel étaient projetés les mots « Cabinet Making 1820-1960", se tenaient face à face un poteau cruciforme en bois et quatre chaises et fauteuils posés sur un piédestal dont un en bois précieux décoré de nacre. L'artiste avait ainsi mis en présence des objets témoins d'une époque directement reliée au passé esclavagiste de la ville de Baltimore, objets conservés, bien à l'abri, dans les entrepôts du musée situé à proximité d'un quartier noir. Il les a mis en scène dans l'espace et dans le temps afin d'amener le spectateur contemporain à une réflexion sur le rapport de l'histoire avec le pouvoir mais aussi sur le rapport entre art et temps.

7 "Ouvrir le musée », comme l'on ouvrirait le temps ou la Vénus de Botticelli, « aussi belle qu'elle est nue [...] aussi reclose, aussi impénétrable qu'elle est belle », pour reprendre les termes de Georges Didi-Huberman (11). Donc «ouvrir le musée » pour en dévoiler l'intériorité, pour sonder cet espace monolithique, lieu du secret où sont enfouis et préservés les trésors de la nation. Pour l'artiste-curateur, cela signifie relever des traces, s'approprier des objets et récolter des témoignages (du personnel du musée, de la communauté locale). Le processus d'élaboration de l'œuvre s'apparente à un travail de mémoire destiné à rendre visible des pans d'histoire occultés et à surmonter la peur du passé qui toujours hante le présent, comme le dit à juste titre Paul Virilio : «à la peur de l'avenir vient à succéder la peur du passé, comme si ce dernier, loin de disparaitre, de s'effacer derrière le présent, continuait à l'encombrer, pire à le contaminer secrètement " (11). Ce travail de "mise en scène» de fragments du passé que Fred Wilson expose au regard du spectateur contemporain se double d'une dimension critique, non seulement 
de l'histoire des États-Unis mais aussi du passé colonial des grandes puissances occidentales, autre thème récurrent dans le travail de cet artiste.

Télescopage de lieux et de temporalités également lorsque l'artiste-ethnologue confronte l'ici et l'ailleurs, le proche et le lointain, le maintenant et l'autrefois, comme dans Anthropologists! Anthropologists! (1991). Ce montage photographique, placé selon un axe vertical, se compose de deux photos (l'une en noir et blanc, datée, l'autre en couleurs, non datée) séparées par un dessin humoristique de Gary Larson (1994). Toute la charge ironique et parodique de l'installation est contenue dans le titre de l'œuvre, lui-même emprunté à la légende enchâssée dans le dessin de Larson.

Dans ce montage chronologique, différentes temporalités sont mises en regard: le temps de l'histoire, le temps du mythe et de l'imaginaire, le temps virtuel et le présent. Dans la première photo placée en haut du dispositif, Wilson s'est approprié une photographie montrant un couple de missionnaires américains envoyés par le Comité Américain des Missions Étrangères en Afrique Occidentale. Sur le cartel, le spectateur peut lire : George and Jewel Schwab in canoe on the Nyong River, Cameroon, c. 1920, photographer unknown. Fortement codée, cette photo emprunte le langage visuel figé avec ses stéréotypes habituels caractéristiques de la photographie coloniale mettant en évidence les rapports dominant/dominé : position assise des colons vêtus de blanc au premier plan, coiffés du chapeau traditionnel et du casque colonial de rigueur ; pose figée ; regard tourné vers le photographe alors qu'à l'arrière sont relégués les indigènes qui pilotent la pirogue. Et pourtant, ces derniers (deux debout, deux assis) qui, à première vue, se fondent dans le paysage, arrêtent le regard. Mystérieuses, leurs silhouettes se détachent sur l'eau paisible du fleuve Nyong et sur les trouées de ciel bleu derrière elles. Dans ce paysage marécageux où algues et branches forment un entrelacs serré, leur reflet dans la surface luisante de l'eau leur donne une matérialité et une présence forte. Telles des statues ou des figures totémiques, elles s'ancrent dans le paysage qu'elles habitent, aux confins de l'eau, de l'air et de la terre. Le choix de l'artiste n'est pas anodin : il a sélectionné une photo coloniale de facture classique certes mais qui néanmoins propose une lecture plus nuancée grâce au travail d'écriture subtil des ombres et de la lumière. Ici, les reflets noirs des corps des indigènes dans l'eau du fleuve confèrent à ces figures longilignes une beauté plastique.

À travers cette photographie, l'artiste souligne ici le discours et la rhétorique d'une autre institution, l'anthropologie traditionnelle: le regard porté sur l'indigène, l'attrait de l'exotisme et le pouvoir des empires coloniaux. Il montre également les potentialités du médium photographique, son pouvoir de fixation du temps, à travers la matérialité de la photo, son grain, la couleur sépia qui creuse la profondeur du temps. La photographie dévoile son utilisation comme outil de captation et pouvoir de domination. En outre, elle signale son pouvoir de rétention (vision d'un passé glorieux) et de suspension du temps. Roland Barthes l'a souligné à plusieurs reprises : «La photographie devient pour moi un médium bizarre, une nouvelle forme d'hallucination: fausse au niveau de la perception, vraie au niveau du temps; une hallucination tempérée en quelque sorte, modeste, partagée [...] image folle, frottée de réel » (177). Sous la photographie, Wilson a ajouté le mot «Myth", comme pour mieux souligner l'imbrication du mythe et de l'histoire et le lien étroit entre mythe et anthropologie, rappelant ainsi les images fantasmées et projetées sur le visage de l'autre intemporel. L'exotisme introduit un décalage et des sentiments ambivalents. Laurent Gervereau explique: "l'exotisme demeure lié à l'impression de possession de la différence [...]. L'exotisme n'est pas géographique. Il est mental. Inévitable conséquence de la distanciation, il participe soit d'un enfermement de 
l'autre dans le regard qu'on lui porte, soit il cherche peu ou prou à générer des ouvertures, des curiosités » (84). En témoignent les nombreuses photographies coloniales au XIX siècle qui ont façonné l'imaginaire collectif occidental. La photographie atteste et permet, outre le rapprochement de l'ailleurs, de l'étrange et de l'exotique, de le rendre familier et de le conserver pour le futur.

La seconde photographie est un portrait d'un jeune Indien de l'état du Mato Grosso au Brésil : Portrait of a Shavante Youth, Aerons, South East Matto Grosso, Brazil, par William Crawford. Celle-ci semble fonctionner selon le procédé inverse, à la fois dans sa construction et dans le choix du sujet. Ici, c'est l'individu nommé et non réduit au statut d'individu anonyme qui est montré. Le choix de cette photo, prise par l'anthropologue américain William Crawford, participe d'un désir de démontage et de déconstruction des codes de la photographie coloniale par l'angle de prise de vue (légère contre-plongée), la position frontale en plan rapproché, le cadrage serré et le rapport de proximité et de complicité avec le photographe. On retrouve le même type de composition avec trois plans successifs que dans la photographie précédente, mais ici la figure est proche tout en étant au deuxième plan, entre la bicyclette et la forêt défrichée à gauche et à droite. Par ailleurs, le jeune amazonien occupe l'espace tout entier de l'image. Il regarde le spectateur, dans une posture frontale, franche et déterminée, exhibant les objets de la modernité, notamment une bicyclette bleue et un poste de radio quelque peu vieillot, médium qui le relie au reste du monde contemporain.

Ce qui est suggéré ici ("The Real » est inscrit sous la photo), c'est le refus du mythe dans un souci de montrer l'ordinaire et le quotidien du point de vue d'un Amazonien, celui qui occupe l'autre espace, le lointain, The Far Side, pour reprendre le titre du dessin de Gary Larson (The Far Side, cartoon by Gary Larson, 1994) sous lequel est noté « Fantasy ». Le dessin humoristique, intercalé entre les deux photographies, fait rupture et sert de contrepoint ironique. On y voit des indigènes affairés à enlever en toute hâte les appareils électroniques et autres objets de la modernité à la vue d'anthropologues venus les étudier. Caricature de l'anthropologue - à la recherche d'indices, de traces d'autrefois menacées de disparaître - dont les méthodes d'investigation passéistes trahissent un regard dominateur et un sens unique de la connaissance. La légende « Fantasy » fait écho à «Myth» et renvoie au rêve exotique, aux scénarii imaginaires par lesquels le sujet (colon) met en scène ses désirs. À l'inverse, comme le laisse entendre la légende, la photographie en couleurs invite à un questionnement sur le réel qui, comme l'explique l'ethno-cinéaste Marc-Henri Piault émane du regard qui «devient peu à peu le constituant d'une réalité sans cesse en train de se faire» (90). En effet, ici l'environnement du jeune indien est à l'arrière-plan et ce qui est mis en exergue, c'est son regard et son attitude qui attestent une réalité multiforme et fluctuante. Par ailleurs, comme nous l'avons dit précédemment, la manipulation du médium photographique signale la présence d'une expérience partagée qui exclut de penser l'image en dehors de ce rapport entre le photographe et le sujet photographié. Citons à nouveau Marc-Henri Piault qui évoque le déplacement du regard de l'anthropologue non plus centré sur l'objet mais se substituant à un échange de regards et un « désir partagé de connaissance » (245).

De la photo coloniale à la photo contemporaine, ce que souligne ce montage, c'est d'abord une réflexion sur le médium photographique qui est le résultat d'une construction selon des paramètres subjectifs, idéologiques et culturels. Il révèle également une disjonction, l'écart temporel et la lisibilité de l'image dans le présent - la collision entre les images du passé et le regard au présent qui active ces représentations. Ainsi face à ces œuvres, le 
spectateur est entraîné dans une sorte de va-et-vient spatial et temporel, parfois déconcertant car suscitant un sentiment d'inquiétante étrangeté. On peut citer à cet égard, et en écho à ce montage, une autre installation de Fred Wilson intitulée The Other Museum (1990) simulant la scénographie du musée ethnologique. Dans une des salles de l'exposition, Colonial Collection montrait une série de masques africains accrochés au mur surplombant une vitrine dans laquelle l'artiste avait placé deux photographies montrant des indigènes rassemblés autour de colons. Sur le bord de chacune des photographies reposait à droite, un crâne humain et à gauche, une mâchoire inférieure, restes de fouilles archéologiques, sortes de memento mori. Au milieu de la vitrine se trouvaient des petites boîtes contenant des insectes et des papillons classés selon les espèces et la taille. Le cartel, placé frontalement, signalait l'origine des objets exposés ( «FRENCH AND BRITISH COLLECTION»). Au mur, des bandes de tissu aux couleurs des drapeaux français et britanniques recouvraient les yeux et/ou la bouche des masques africains. Dans l'installation Bwana Memorial Gallery of African Art, deux masques africains étaient animés d'une vidéo. Sous l'effet d'un projecteur, le masque se mettait en mouvement, déclenchait un clignement des yeux et un mouvement des lèvres. De cette figure mécanique à la Disney, selon les mots de l'artiste (Museum Studies 400 notes) s'échappait une voix qui répétait : « Don't just look at me; speak to me. I'm still alive », sorte de voix d'outre-tombe qui brisait le silence de la contemplation. L'autre masque dissimulait une vidéo à l'emplacement des yeux qui relatait des nouvelles de la guerre entre l'armée britannique et les Zoulous. À travers le masque, objet fétiche symbole d'un temps circulaire associé aux rites et rituels, étaient condensées et imbriquées plusieurs temporalités : l'histoire de l'empire britannique, l'histoire des peuples africains et l'histoire de l'objet luimême (l'origine du masque, son devenir et le regard porté par le spectateur contemporain).

Ainsi, grâce à l'interaction de plusieurs médiums - visuel, sonore, verbal - se tisse un espace dialogique à l'intérieur duquel le spectateur est sans cesse sollicité et invité à s'interroger sur le choix des objets, sur le lien entre passé, présent et avenir, et sur sa propre perception. Dans l'enchevêtrement d'éléments hétérogènes se donne à lire une écriture de la collision qui révèle la puissance de chaque matériau, ici le pouvoir de la voix que l'on pourrait dire atemporelle, voix qui interpelle le spectateur dans le présent de l'observation.

\section{À rebours/remontage du temps}

15 C'est donc souvent à un parcours à rebours qu'est convié le spectateur, parcours qui consiste à remonter le temps, « remonter » dans le double sens du terme, de "retour en arrière » et dans le sens physique et artistique de "recomposition, recréation ». Pour l'artiste, ce travail s'apparente à une relecture de l'histoire à partir d'éléments du passé dans leur articulation au présent toujours à construire. Telle est l'idée sous-jacente de l'œuvre multimédia créée par Fred Wilson pour la biennale de Venise en 2003 (autre institution, haut lieu du marché de l'art contemporain où Robert Colescott a été le premier artiste noir à représenter les USA en 1997), intitulée Speak of Me as I Am, dont le titre est extrait de la dernière tirade d'Othello (5.2.336-39 :

No more of that. I pray you, in your letters

When you shall these unlucky deeds relate, 
Speak of me as I am. Nothing extenuate,

Nor set down aught in malice.

16 À nouveau, il est important de préciser la démarche conceptuelle et tout ce qui a précédé la réalisation de l'œuvre : l'observation et le travail in situ, la recherche menée dans les archives, dans les musées, le contact avec la population locale. Ce qui intéressait Wilson, c'était l'histoire de Venise et les rôles qu'ont joué les Noirs dans cette ville, appelée «la cité de la Renaissance» ou encore "la cité des immigrants» (Hassan 56), ville cosmopolite tout à la fois attirante et complexe, comme en témoignent les nombreux écrits d'historiens, les tableaux de l'époque et l'architecture. Wilson a établi des liens entre cette période lointaine riche et féconde et la ville de Venise aujourd'hui, marquée de tensions sociales, de violence et de précarité. En déambulant dans les rues de la cité italienne, il a été frappé par la forte présence d'immigrés clandestins, une population africaine, originaire essentiellement du Sénégal, des vendeurs à la sauvette, traqués par les services d'ordre, et qui proposent aux touristes des sacs de marque de contrefaçon.

L'aboutissement de son projet a été la réalisation d'une œuvre protéiforme, créée à partir d'un ensemble d'éléments du passé juxtaposés et associés à des fragments puisés dans le monde contemporain mettant en scène des hétérogénéités et des anachronismes. Cette œuvre rhizomique fluide et fluctuante faite de multiples ramifications s'étendait à l'intérieur du pavillon américain et débordait sur l'espace public à l'extérieur. La performance proposée au spectateur le jour de l'ouverture de la biennale en est un exemple. Wilson avait engagé un jeune Sénégalais qui, devant le pavillon américain, vendait des sacs à main que lui-même avait fabriqués et dont le tissu rappelait les costumes chamarrés des mannequins à l'intérieur du bâtiment. En retrait, il observait ce qui ressemblait à une scène typique d'un jour ordinaire lorsque des agents de l'ordre délogent des marchands illicites. D'emblée, le spectateur était confronté aux réalités du monde contemporain, avec ses aléas et ses turbulences. Pour cet événement, il a donc composé une œuvre interactive et polyphonique où s'affrontaient des objets et se faisaient entendre des énonciations plurielles et discordantes. Le tout était constitué d'un matériau hétéroclite fait d'objets de sa collection ou appropriés sur place, et d'un matériau vivant (la population locale et l'artiste lui-même interagissant avec le public dans le pavillon et dans la rue).

Son travail s'articulait autour de deux axes complémentaires, l'un centré sur la figure du Maure, figure de l'aliénation, de l'étranger dans la cité, et l'autre sur le trope (dans son sens originaire d' " ornement ») de la noirceur. Cette intention était affichée avant même d'entrer dans le pavillon américain, monument de style palladien flanqué de deux cariatides noires. En effet, sur son chemin, le spectateur pouvait voir de longs drapés noirs posés sur des statues gréco-romaines.

Entrer dans le pavillon américain, c'était pénétrer dans le monde de la représentation, de la mise en scène et des apparences. C'était aussi se sentir submergé par un flot d'images visuelles et sonores qui avaient une résonance particulière car portée par une inquiétante étrangeté. Parmi ces éléments, on peut énumérer toute une série d'objets anachroniques : à l'entrée, l'imposant lustre de style baroque fait en verre noir de Murano se détachant sur un mur jaune criard, une couleur discordante rappelant, d'une part, l'intérieur des églises et des palais de la ville (les fonds dorés des icônes et des tableaux) et, d'autre part, dans le contexte américain troublé des années 60 , le système de busing et de ségrégation raciale. Une fois le seuil franchi, dans une niche devant une fenêtre oblitérée, également 
de couleur jaune, le spectateur se trouvait face à une statue dorée représentant un page tenant un plateau. Sa tête était affublée d'un énorme globe montrant le continent africain. Le même contraste se retrouvait à l'intérieur où trônaient des figurines (en verre translucide portant des bouteilles de parfum) et des diablotins noirs dupliquant les objets vendus aux touristes dans les hôtels et magasins de Venise ou encore des mannequins noirs grandeur nature (placés dans une vitrine), habillés en costume d'époque (dont la figure de Balthazar), personnages tout droit sortis des peintures de Véronèse, de Carpaccio ou de Mantegna.

Cependant, comme à l'accoutumée chez Fred Wilson, l'inconfort s'installait très vite. La scène se désagrégeait au contact du présent y faisant irruption: présence d'une figurine noire tenant un chandelier dont le corps était pris dans un entrelacs de cordons bleus et rouges reliés à des bouteilles d'explosifs. Une autre installation au titre évocateur, Turbulence II, arrêtait le regard du spectateur. Cette installation formait un espace fermé une pièce dans la pièce - dont le sol et les murs étaient tapissés d'un motif carrelé noir et blanc. Deux miroirs entièrement noirs étaient accrochés au mur. Malgré son titre, cette pièce, de facture plus abstraite évocatrice de l'art optique, était aussi plus intime avec l'urne noire posée au sol dans laquelle se trouvaient un lit en miniature, une tasse à café, des magazines, sorte de clin d'œil à Marcel Duchamp et sa célèbre Boîte-en-Valise contenant des répliques miniatures d'œuvres. Sur un cartel devant l'urne était écrit "Safe Haven ", mots trompeurs car la répétition du motif abstrait noir et blanc suscitait une sensation de vertige, une impression déstabilisante corroborée par l'autre installation au titre ironique située à proximité de celle-ci, September Dream. Là, sur quatre écrans vidéos non synchronisés (séquences de 7 minutes) apparaissaient des scènes de meurtre d'Othello jouées par des acteurs blancs et montrées à rebours, du meurtre aux scènes d'amour. Ces scènes répétées en boucle renforçaient le sentiment de vertige visuel et auditif et pouvaient être perçues comme la matérialisation d'un temps démesuré et incontrôlable. Elles étaient accompagnées d'extraits d'opéra, dont l'opéra de Verdi, une manière pour l'artiste de renverser le cours de l'histoire, en réponse à la tragédie cauchemardesque du 11 septembre, comme l'explique Fred Wilson dans un entretien (Goncharov 24). L'artiste a donc utilisé la figure d'Othello comme trope de la différence et de l'aliénation, figure de la marge qui se répète d'un continent à un autre : la séquence en boucle et les scènes de violence montrées au ralenti introduisent l'idée d'un temps cyclique, du confinement et de la mort.

21 Parmi les autres pièces, de nature et de facture différente mais toujours en résonance avec le trope de la noirceur, on peut mentionner Drip, Drop, Plop, des « larmes » en verre noir donnant l'impression de couler le long d'un mur blanc avant de se déposer sur le sol. Le titre aux sons onomatopéiques ouvre la pièce à plusieurs lectures. C'est tout d'abord la figure d'Othello qui apparait en filigrane, sa voix qui se fait entendre dans le même passage précédant son suicide (5.2.339-47) :

Then must you speak

Of one that loved not wisely, but too well;

of one not easily jealous, but being wrought,

Perplexed in the extreme; of one whose hand,

Like the base Judean, threw a pearl away

Richer than all his tribe; of one whose subdued eyes,

Albeit unusèd to the melting mood,

Drops tears as fast as the Arabian trees

Their med'cinable gum. [...] 

Elles sont aussi les perles associées à la beauté de Desdémone ( threw a pearl away »), perles de désir évocatrices de la photographie surréaliste Les Larmes (1932-33) de Man Ray montrant l'œil d'une femme, en pleurs. En outre, elles s'associent à la notion d'africanité, comme le suggère la référence à la gomme arabique s'écoulant des arbres (« Drops tears as fast as the Arabian trees/Their med'cinable gum »). Pour Fred Wilson, le lien entre Drip, Drop, Plop et l'africanité est évident mais ce concept ne peut se réduire au seul continent africain. L'africanité est une question fluide et mouvante, toujours à reconstruire. Le spectateur peut aussi interpréter cette substance noire comme métaphore du rejet et de l'aliénation engendrés par la prolifération de stéréotypes et de clichés (Goncharov 24). Cette impression est corroborée par le photomontage en couleurs également visible dans une des salles du pavillon. Celui-ci était constitué d'une série de photos de jeunes Sénégalais mêlées à des fragments de reproductions de tableaux représentant des Noirs dans des postures et des contextes différents. Au dispositif photographique, Fred Wilson avait ajouté une vidéo, médium à travers lequel il donnait la parole à ces immigrés clandestins, marchands ambulants que les Vénitiens appellent les $v u$ compra (expression hybride utilisée par les premiers vendeurs noirs durant leur négoce), autre manière pour l'artiste de confronter le milieu protégé de l'art aux incertitudes du monde contemporain.

On le voit, Fred Wilson aime jouer sur les anachronismes, les décalages et heurts temporels en tissant une écriture où le passé fait sans cesse irruption dans le présent, où il se force un passage dans le monde contemporain. Pour cet artiste africain américain, ce travail sur les traces du temps en lien avec les structures institutionnelles est aussi un travail de mémoire, contre l'oubli, contre la violence et les cicatrices du temps.

L'œuvre bouleverse les repères et la perception du spectateur, par le mélange des genres, les stratégies de répétition et de juxtaposition, les ruptures et les discordances visuelles et temporelles. En livrant une œuvre dans laquelle se croisent et se confrontent des temporalités hétérogènes, Fred Wilson souligne ainsi les excès et les paradoxes du temps.

\section{BIBLIOGRAPHIE}

Barthes, Roland. La Chambre claire. Paris : Éditions du Seuil, 1980.

Didi-Huberman, Georges. Ouvrir Vénus. Paris : Gallimard, 1999.

Elliott, Dorinda. “Sleeping Beauty”. Newsweek (September 11, 1995): 58-59.

Foucault, Michel. Surveiller et punir : naissance de la prison (1975). Paris : Gallimard, 2002.

Gervereau, Laurent. Les Images qui mentent : histoire du visuel au XXe siècle. Paris : Éditions du Seuil, 2000.

Goncharov, Kathleen \& Fred Wilson. "Interview”. Jane Farver, Paul Kaplan, Kathleen Goncharov \& Hassan Salah (eds.). Speak of Me as I Am. Cambridge, MA: MIT, 2003, 20-25.

Polysèmes, $17 \mid 2017$ 
Gorin, Lisa G. "Mining the Museum: Artists Look at Museums, Museums Look at Themselves". Carbonell Bettina Messias (ed.). Museum Studies. Malden, MA: Blackwell, 2004.

Hassan, Salah. « Parle de moi comme je suis. Représentation d'une cité hybride ». Malaquais Dominique \& Roland Marchal (dir.). « Cosmopolis : de la ville, de l'Afrique et du monde ». Politique Africaine 100 (décembre 2005 / janvier 2006) : 54-64.

Piault, Marc-Henri. Anthropologie et Cinéma. Passage à l'image, passage par l'image. Paris : Nathan, 2000.

Shakespeare, William. Othello. New York : Signet Classic, 1963.

Virilio, Paul. Un Paysage d'événements. Paris : Galilée, 1996.

\section{NOTES}

1. Pour Fred Wilson, ce projet avait pour objectif de mettre en lumière le passé tourmenté de West Salem où la communauté noire fut tour à tour intégrée puis rejetée par la communauté morave venue s'installer en 1772. Le cimetière, pour la population noire et non-Morave, relégué à la sortie de la ville, est emblématique des tensions raciales et de l'atmosphère qui régnait dans cette petite localité de Caroline du nord. Construite d'abord en bois, l'église fut en 1861 agrandie et reconstruite en briques. Fermée pendant presque quarante ans, elle fut restaurée en 1989.

2. D'autres exemples peuvent être cités qui ressortissent de la même démarche muséale, comme la controverse suscitée par une jeune équipe de chercheurs désireux d'ouvrir les archives de la section d'ethnographie au Musée d'Afrique centrale à Bruxelles en 1995. Ce projet avait fait couler beaucoup d'encre car subitement tout le passé colonial de la Belgique refaisait surface. Voir à ce sujet l'article de Dorinda Elliott, "Sleeping Beauty", Newsweek (September 11, 1995): 58-59.

\section{RÉSUMÉS}

On peut dire que la spécificité du travail de Fred Wilson, artiste plasticien né à New York en 1954, réside dans les collisions et télescopages temporels que ses œuvres, et en particulier ses installations, mettent en scène. Cet artiste africain américain est connu essentiellement pour ses pratiques curatoriales réalisées dès la fin des années 80 , notamment son travail d'investigation et de fouille dans les archives et collections des musées, ces monuments dépositaires de la mémoire d'une nation dont il parodie le langage et la rhétorique. Préoccupé par l'histoire, la mémoire et les questions relatives à l'identité du peuple africain américain, Fred Wilson questionne le monde contemporain à travers une pratique artistique fondée sur un mouvement de va-et-vient constant entre passé et présent et à travers un dispositif qui met en lumière à la fois une esthétique de la collision (choc de civilisations et de cultures, confrontation art occidental/art oriental, art majeur/art mineur) et de l'hybridité des genres, des formes et de matières. Ces préoccupations d'ordre historique, géographique, social, culturel et formel s'accompagnent d'une réflexion et d'un jeu sur les codes de représentation. Dans ses installations créées à l'intérieur et à l'extérieur de lieux publics et privés, Fred Wilson montre comment les ruptures et disjonctions temporelles contribuent à susciter un sentiment d'étrangeté et à amener le 
spectateur à s'interroger sur sa propre perception des œuvres. Le présent article propose d'examiner les collisions et télescopages temporels mis en œuvre par l'artiste dans ses installations, à travers la juxtaposition, l'entrecroisement et la confrontation entre passé et présent.

The singularity of Fred Wilson's art (born in New York in 1954) lies mainly in the temporal collisions and clashes embedded in his practice and in particular his installations. This African American artist is well-known for the curatorial experiments he has carried out since the late 1980s in the collections and archives of museums, those monuments - memory deposits of a nation - whose language and rhetoric he parodies. Concerned with history, memory and questions pertaining to African American identity, Fred Wilson interrogates our contemporary world through an art practice based on a constant back and forth movement between past and present and through an interactive device that highlights an aesthetics of collision (historical and cultural, western vs. eastern art, major vs. minor art) and hybridity (of genre, form, and material). Along with those historical, geographic, social, cultural, and formal concerns, his work evidences a reflection and play on representational codes. In his indoor or outdoor installations, be they in private or public places, Fred Wilson shows how temporal passages, breaks and disjunctions give rise to a feeling of strangeness and lead the spectator to question his/her own perception of works. The purpose of this article is to examine how the collisions and temporal clashes operate in the artist's installations, through the process of juxtaposing, weaving together, and confronting past and present.

\section{INDEX}

Keywords : visual arts, installation, institutional critique, hybridity, perception, representation, anthropology

Mots-clés : arts plastiques, installation, critique institutionnelle, hybridité, perception, représentation, anthropologie

\section{AUTEURS}

\section{CLAUDINE ARMAND}

Claudine Armand est maître de conférences à l'Université de Lorraine, Nancy. Son aire de recherche se situe dans le champ de l'intersémioticité et de l'intermédialité. Ses travaux portent essentiellement sur des artistes américains qui explorent des médias hétérogènes et multiplient les liens et passerelles entre les arts visuels et le langage verbal. Elle est l'auteur d'un catalogue d'exposition, Anne Ryan : Collages (Musée d'Art Américain de Giverny, Terra Foundation, 2001) et de plusieurs articles sur des artistes modernes et contemporains. Elle a codirigé un ouvrage intitulé Ancrages/Passages (2006), un livre sur le mythe de Prométhée dans les arts et la littérature, Créatures et créateurs de Prométhée (2010), sur Londres-New York : Échanges, influences, croisements dans les arts et la littérature (2012) et sur l'interdisciplinarité, Enjeux et Positionnements de l'interdisciplinarité/Positioning Interdisciplinarity (2014). Elle poursuit ses recherches sur les pratiques pluridisciplinaires d'artistes contemporains et travaille actuellement sur la rédaction d'un ouvrage sur la voix dans l'art contemporain africain américain et amérindien. 